\title{
Global existence for nonlinear wave equation
}

\author{
Svetlin G. Georgiev \\ Communicated by Y. Charles Li, received June 20, 2010.
}

\begin{abstract}
In this work we propose a new approach for investigating the local and global existence of solutions of nonlinear wave equations. This approach gives new results.
\end{abstract}

\section{Contents}

1. Introduction 207

2. Proof of Main Result 208

$\begin{array}{ll}\text { References } & 216\end{array}$

\section{Introduction}

Nonlinear wave equations are introduced as models of physical phenomena in general relativity, mechanics and relativistic field theories. The main tools for studying these models are the classical representation formula of solutions using the fundamental solution, due to d'Alambert and energy estimates. These estimates were used in the works [3], [5], [6] (and references therein) to establish local existence and uniqueness of solutions of these phenomena. Global existence for these equations with Lipschitz nonlinearities are based on energy estimates(see [3], [5], [6] and references therein). For nonlinearities with restrictive growth are used Strichartz - type estimates for local existence of strong finite energy solutions (see [5], [6] and references therein). Global existence for subcritical problems is obtained using energy conservation laws. Global existence for critical problems follows from a nonconcentration lemma derived from the scaling identity(see [6] and references therein).

In this work we propose a new approach for investigating of these problems. This approach gives new results. It is based on a new integral representation of the

2000 Mathematics Subject Classification. 35L10, 35L70.

Key words and phrases. Nonlinear wave equation, local existence, global existence. 
solutions. We focus our attention on the Cauchy problem

$$
\begin{gathered}
u_{t t}-\frac{n-1}{r} u_{r}-u_{r r}=u_{t}^{p}, \quad t \geq 0, r \geq r_{0}, \\
u(0, r)=u_{0}(r), \quad u_{t}(0, r)=u_{1}(r), \quad r \geq r_{0},
\end{gathered}
$$

where $r_{0}>0$ is fixed, $n \geq 2$ is fixed, $r=|x|=\sqrt{x_{1}^{2}+x_{2}^{2}+\cdots+x_{n}^{2}}, p \geq 0$ is fixed.

Let $Q>0$ is arbitrary chosen and fixed. Our main assumption for the initial data $u_{0}(r)$ and $u_{1}(r)$ are

(H1) $u_{0}(r) \in \mathcal{C}^{2}\left(\left[r_{0}, \infty\right)\right), u_{1}(r) \in \mathcal{C}^{1}\left(\left[r_{0}, \infty\right)\right),\left|u_{0}(r)\right| \leq Q,\left|u_{1}(r)\right| \leq Q$ for every $r \geq r_{0}$.

Our main result is

THEOREM 1.1. Let $n \geq 2$ be fixed, $p \geq 0$ be fixed, $Q>0$ be fixed, $r_{0}>0$ be fixed, the initial data $u_{0}, u_{1}$ satisfy the hypothesis $(H 1)$. Then the Cauchy problem (1.1), (1.2) has a solution $u$ for which $u \in \mathcal{C}^{2}\left([0, \infty), \mathcal{C}^{2}\left(\left[r_{0}, \infty\right)\right)\right)$.

We note that if $u_{0}$ or $u_{1}$ is not udentically equal to zero then since $u(t, r) \in$ $\mathcal{C}^{2}\left([0, \infty), \mathcal{C}^{2}\left(\left[r_{0}, \infty\right)\right)\right)$ we have that $u(t, r)$ is nontrivial.

Since we search a solution $u(t, r) \in \mathcal{C}^{2}\left([0, \infty), \mathcal{C}^{2}\left(\left[r_{0}, \infty\right)\right)\right)$ of the problem $(1.1)$, (1.2) we have not a need of bounded conditions in the formulation of the considered problem because if $u(t, r) \in \mathcal{C}^{2}\left([0, \infty), \mathcal{C}^{2}\left(\left[r_{0}, \infty\right)\right)\right)$ we have $u(t, r)_{\left.\right|_{r=r_{0}}}=$ $u\left(t, r_{0}\right), u_{t}(t, r)_{\left.\right|_{r=r_{0}}}=u_{t}\left(t, r_{0}\right), u_{t t}(t, r)_{\left.\right|_{r=r_{0}}}=u_{t t}\left(t, r_{0}\right), u_{r}(t, r)_{\left.\right|_{r=r_{0}}}=u_{r}\left(t, r_{0}\right)$, $u_{r r}(t, r)_{\mid r=r_{0}}=u_{r r}\left(t, r_{0}\right)$ for every $t \in[0, \infty)$.

Obviously the problems for local existence, global existence, blow up for the equation (1.1) are connected with the values of $p$ and $n$. This connectien is due of the inegral representations which are used for their investigation. Here we use new inetgral representation which show us that these problems are connected with the integral represenation and they are not connected with the values of $p$ and $n$. We note that our condition for $p$ is $p \geq 0$ and for $n: n \geq 2$.

We will compare our result with well known result. Of course, there are many other articles which are connected with the problem (1.1), (1.2), our aim is to give one example with a well known result which illustrates the power of our approach. When $p=$ const $>2, n=3, u_{0}$ and $u_{1}$ have compact support, in [7] is obtained the global in time existence in weighted $L^{\infty}$ - spaces of a classical solution of the problem (1.1), (1.2).

This new nice result is due to our new integral representation.

In the next section we will prove our main result.

\section{Proof of Main Result}

Let $\epsilon=\frac{1}{100}$. Let also $A>0$ is constant for which

$$
\frac{1}{2} A^{2} Q^{p-1}+\frac{1}{2}+\frac{1}{2} A^{2}+\frac{1}{2} \frac{n-1}{r_{0}^{2}} A^{2}+2 A^{2}+\frac{1}{2} A \frac{n-1}{r_{0}} \leq(1-\epsilon),
$$

in the case $p>0$ and

$$
\frac{1}{2}+\frac{1}{2} A^{2}+\frac{1}{2} \frac{n-1}{r_{0}^{2}} A^{2}+2 A^{2}+\frac{1}{2} A \frac{n-1}{r_{0}} \leq(1-\epsilon)
$$

in the case $p=0$. Such choice is possible since we can choose $A<1$ enough small. 
Firstly we will prove that the Cauchy problem

$$
\begin{gathered}
u_{t t}-\frac{n-1}{r} u_{r}-u_{r r}=u_{t}^{p}, \quad t \in\left[0, \frac{1}{2}\right], r \in\left[r_{0}, r_{0}+A\right], \\
u(0, r)=u_{0}(r), \quad u_{t}(0, r)=u_{1}(r), \quad r \in\left[r_{0}, r_{0}+A\right]
\end{gathered}
$$

has a solution which belongs to the space $\mathcal{C}^{2}\left(\left[0, \frac{1}{2}\right], \mathcal{C}^{2}\left(\left[r_{0}, r_{0}+A\right]\right)\right)$. For this purpose we will use fixed point arguments. Therefore we have a need of suitable operator whose fixed point is a solution to the Cauchy problem (2.1), (2.2) and belongs to the space $\mathcal{C}^{2}\left(\left[0, \frac{1}{2}\right], \mathcal{C}^{2}\left(\left[r_{0}, r_{0}+A\right]\right)\right)$.

Let us consider the integral operator

$$
\begin{aligned}
& L_{11}(u)=\int_{0}^{t} \int_{0}^{s} \int_{r}^{A} \int_{y}^{A} u_{t}^{p} d z d y d \tau d s \\
& +\int_{0}^{t} \int_{0}^{s} u(\tau, r) d \tau d s+\int_{0}^{t} \int_{0}^{s} \int_{r}^{A} \int_{y}^{A} \frac{n-1}{z^{2}} u(\tau, z) d z d y d \tau d s+t \int_{r}^{A} \int_{s}^{A} u_{1}(y) d y d s+ \\
& +\int_{r}^{A} \int_{s}^{A} u_{0}(y) d y d s-\int_{r}^{A} \int_{s}^{A} u(t, y) d y d s-\int_{0}^{t} \int_{0}^{s} \int_{r}^{A} \frac{n-1}{y} u(\tau, y) d y d \tau d s+u(t, r) .
\end{aligned}
$$

Let $u \in \mathcal{C}^{2}\left(\left[0, \frac{1}{2}\right], \mathcal{C}^{2}\left(\left[r_{0}, r_{0}+A\right]\right)\right)$ is a fixed point to the operator $L_{11}$, i.e. $L_{11}(u)=$ $u$. Then, from $(2.3)$, we have

$(2.4)$

$$
\begin{aligned}
& \int_{0}^{t} \int_{0}^{s} \int_{r}^{A} \int_{y}^{A} u_{t}^{p} d z d y d \tau d s+\int_{0}^{t} \int_{0}^{s} u(\tau, r) d \tau d s+ \\
& +\int_{0}^{t} \int_{0}^{s} \int_{r}^{A} \int_{y}^{A} \frac{n-1}{z^{2}} u(\tau, z) d z d y d \tau d s+t \int_{r}^{A} \int_{s}^{A} u_{1}(y) d y d s+ \\
& +\int_{r}^{A} \int_{s}^{A} u_{0}(y) d y d s-\int_{r}^{A} \int_{s}^{A} u(t, y) d y d s-\int_{0}^{t} \int_{0}^{s} \int_{r}^{A} \frac{n-1}{y} u(\tau, y) d y d \tau d s=0
\end{aligned}
$$

Now we differentiate in $t$ the last equality and we obtain

$$
\begin{aligned}
& \int_{0}^{t} \int_{r}^{A} \int_{y}^{A} u_{t}^{p} d z d y d s+\int_{0}^{t} u(\tau, r) d \tau+ \\
& +\int_{0}^{t} \int_{r}^{A} \int_{y}^{A} \frac{n-1}{z^{2}} u(\tau, z) d z d y d \tau+\int_{r}^{A} \int_{s}^{A} u_{1}(y) d y d s- \\
& -\int_{r}^{A} \int_{s}^{A} u_{t}(t, y) d y d s-\int_{0}^{t} \int_{r}^{A} \frac{n-1}{y} u(\tau, y) d y d \tau=0
\end{aligned}
$$

Again we differentiate in $t$ and we obtain

$$
\begin{aligned}
& \int_{r}^{A} \int_{y}^{A} u_{t}^{p} d z d y+u(t, r)+\int_{r}^{A} \int_{y}^{A} \frac{n-1}{z^{2}} u(t, z) d z d y- \\
& -\int_{r}^{A} \int_{s}^{A} u_{t t}(t, y) d y d s-\int_{r}^{A} \frac{n-1}{y} u(t, y) d y=0 .
\end{aligned}
$$

Now we differentiate in $r$

$$
\begin{aligned}
& -\int_{r}^{A} u_{t}^{p} d z+u_{r}-\int_{r}^{A} \frac{n-1}{y^{2}} u(t, y) d y+ \\
& +\int_{r}^{A} u_{t t}(t, y) d y+\frac{n-1}{r} u(t, r)=0
\end{aligned}
$$

again we differentiate in $r$

$$
u_{t}^{p}=-u_{r r}+u_{t t}(t, r)-\frac{n-1}{r} u_{r}(t, r) .
$$


Now we put $t=0$ in (2.4) and we obtain

$$
\int_{r}^{A} \int_{s}^{A} u_{0}(y) d y d s-\int_{r}^{A} \int_{s}^{A} u(0, y) d y d s=0 .
$$

After we differentiate the last equality twice in $r$ we obtain

$$
u_{0}(r)=u(0, r)
$$

Now we put $t=0$ in the equality (2.5) and we obtain

$$
\int_{r}^{A} \int_{s}^{A} u_{1}(y) d y d s-\int_{r}^{A} \int_{s}^{A} u_{t}(0, y) d y d s=0,
$$

after we differentiate twice in $r$ we obtain

$$
u_{1}(r)=u_{t}(0, r) .
$$

Consequently, if $u(t, r)$ is a fixed point to the operator $L_{11}$, we have that $u(t, r)$ is a solution to the Cauchy problem (2.1), (2.2).

To prove that the operator $L_{11}$ has a fixed point we will use the following fixed point theorem

Theorem 2.1. (see [8], Corrolary 2.4, pp. 3231) Let $X$ be a nonempty closed convex subset of a Banach space $Y$. Suppose that $T$ and $S$ map $X$ into $Y$ such that

(i) $S$ is continuous, $S(X)$ resides in a compact subset of $Y$;

(ii) $T: X \longrightarrow Y$ is expansive and onto.

Then there exists a point $x^{\star} \in X$ with $S x^{\star}+T x^{\star}=x^{\star}$.

Here we will use the following definition for expansive operator.

Definition. (see [8], pp. 3230) Let $(X, d)$ be a metric space and $M$ be a subset of $X$. The mapping $T: M \longrightarrow X$ is said to be expansive, if there exists a constant $h>1$ such that

$$
d(T x, T y) \geq h d(x, y) \quad \forall x, y \in M .
$$

We define the sets $M_{11}$ and $N_{11}$ as equicontinuous subsets of

$$
\begin{gathered}
\tilde{M}_{11}=\left\{u(t, r) \in \mathcal{C}^{2}\left(\left[0, \frac{1}{2}\right], \mathcal{C}^{2}\left(\left[r_{0}, r_{0}+A\right]\right)\right),|u(t, r)| \leq Q,\left|u_{t}(t, r)\right| \leq Q,\right. \\
\left.\forall t \in\left[0, \frac{1}{2}\right], r \in\left[r_{0}, r_{0}+A\right]\right\}, \\
\tilde{N}_{11}=\left\{u(t, r) \in \mathcal{C}^{2}\left(\left[0, \frac{1}{2}\right], \mathcal{C}^{2}\left(\left[r_{0}, r_{0}+A\right]\right)\right),|u(t, r)| \leq(1+\epsilon) Q,\right. \\
\left.\left|u_{t}(t, r)\right| \leq(1+\epsilon) Q, \forall t \in\left[0, \frac{1}{2}\right], r \in\left[r_{0}, r_{0}+A\right]\right\},
\end{gathered}
$$

respectively, in these sets we define a norm as follows

$$
\|u\|_{2}=\sup \left\{|u(t, r)|,\left|u_{t}(t, r)\right| \quad t \in\left[0, \frac{1}{2}\right], r \in\left[r_{0}, r_{0}+A\right]\right\} .
$$

The sets $M_{11}, N_{11}$ are completed normed spaces with this norm, also the set $M_{11}$ is a convex subset of $N_{11}$.

For the operator $L_{11}$ we will use the following representation

$$
L_{11}(u)=T_{11}(u)+S_{11}(u),
$$


where

$$
\begin{aligned}
& T_{11}(u)=(1+\epsilon) u \\
& S_{11}(u)=-\epsilon u+\int_{0}^{t} \int_{0}^{s} \int_{r}^{A} \int_{y}^{A} u_{t}^{p} d z d y d \tau d s \\
& +\int_{0}^{t} \int_{0}^{s} u(\tau, r) d \tau d s+\int_{0}^{t} \int_{0}^{s} \int_{r}^{A} \int_{y}^{A} \frac{n-1}{z^{2}} u(\tau, z) d z d y d \tau d s+t \int_{r}^{A} \int_{s}^{A} u_{1}(y) d y d s+ \\
& +\int_{r}^{A} \int_{s}^{A} u_{0}(y) d y d s-\int_{r}^{A} \int_{s}^{A} u(t, y) d y d s- \\
& -\int_{0}^{t} \int_{0}^{s} \int_{r}^{A} \frac{n-1}{y} u(\tau, y) d y d \tau d s .
\end{aligned}
$$

Lemma 2.2. The operator $T_{11}: M_{11} \longrightarrow N_{11}$ is an expansive operator and onto.

Proof. Firstly we will prove that

$$
T_{11}: M_{11} \longrightarrow N_{11} \text {. }
$$

Really, let $u \in M_{11}$ is arbitrary chosen and fixed. Then $u \in \mathcal{C}^{2}\left(\left[0, \frac{1}{2}\right], \mathcal{C}^{2}\left(\left[r_{0}, r_{0}+\right.\right.\right.$ $A]))$, from here $(1+\epsilon) u \in \mathcal{C}^{2}\left(\left[0, \frac{1}{2}\right], \mathcal{C}^{2}\left(\left[r_{0}, r_{0}+A\right]\right)\right)$, therefore $T_{11}(u) \in \mathcal{C}^{2}\left(\left[0, \frac{1}{2}\right], \mathcal{C}^{2}\left(\left[r_{0}, r_{0}+A\right]\right)\right)$. Also, from $u \in M_{11}$ follows that $|u(t, r)| \leq$ $Q,\left|u_{t}(t, r)\right| \leq Q$ for every $t \in\left[0, \frac{1}{2}\right], r \in\left[r_{0}, r_{0}+A\right]$. From here follows that $\left|T_{11}(u)(t, r)\right| \leq(1+\epsilon) Q,\left|\left(T_{11}(u)\right)_{t}(t, r)\right| \leq(1+\epsilon) Q$ for every $t \in\left[0, \frac{1}{2}\right]$ and every $r \in\left[r_{0}, r_{0}+A\right]$. Consequently $T_{11}(u) \in N_{11}$ from where we conclude that $T_{11}: M_{11} \longrightarrow N_{11}$.

Let now $u, v \in M_{11}$ are arbitrary chosen and fixed. Then, from the defintion for norm in $N_{11}, M_{11}$ we obtain

$$
\left\|T_{11}(u)-T_{11}(v)\right\|_{2}=(1+\epsilon)\|u-v\|_{2},
$$

then $T_{11}: M_{11} \longrightarrow N_{11}$ is an expansive operator with constnat $h=1+\epsilon$.

Now we will see that the operator $T_{11}: M_{11} \longrightarrow N_{11}$ is onto. Let $v \in N_{11}$ is arbitrary chosen and fixed. Then $|v(t, r)| \leq(1+\epsilon) Q,\left|v_{t}(t, r)\right| \leq(1+\epsilon) Q$ for every $t \in\left[0, \frac{1}{2}\right]$ and every $r \in\left[r_{0}, r_{0}+A\right]$. Let $u=\frac{v}{1+\epsilon}$. Then $u \in \mathcal{C}^{2}\left(\left[0, \frac{1}{2}\right], \mathcal{C}^{2}\left(\left[r_{0}, r_{0}+\right.\right.\right.$ $A]))$ and $|u(t, r)| \leq Q,\left|u_{t}(t, r)\right| \leq Q$ for every $t \in\left[0, \frac{1}{2}\right]$ and every $r \in\left[r_{0}, r_{0}+A\right]$. Consequently $u \in M_{11}$ and the operator $T_{11}: M_{11} \longrightarrow N_{11}$ is onto.

LEMMA 2.3. We have

$$
S_{11}: M_{11} \longrightarrow M_{11}
$$

is continuous and $M_{11}$ is a compact subset of $N_{11}$.

Proof. Firstly we will prove that

$$
S_{11}: M_{11} \longrightarrow M_{11} \text {. }
$$

Let $u \in M_{11}$ is arbitrary chosen and fixed. Then, using that $|u|,\left|u_{t}\right| \leq Q, r \in$ $\left[r_{0}, r_{0}+A\right], t \in\left[0, \frac{1}{2}\right]$, and the conditions for the constant $A$ (see the beginning of 
the proof), we have

$$
\begin{aligned}
& \left|S_{11}(u)\right| \leq \epsilon|u|+\int_{0}^{t} \int_{0}^{s} \int_{r}^{A} \int_{y}^{A}\left|u_{t}^{p}\right| d z d y d \tau d s \\
& +\int_{0}^{t} \int_{0}^{s}|u(\tau, r)| d \tau d s+\int_{0}^{t} \int_{0}^{s} \int_{r}^{A} \int_{y}^{A} \frac{n-1}{z^{2}}|u(\tau, z)| d z d y d \tau d s+t \int_{r}^{A} \int_{s}^{A}\left|u_{1}(y)\right| d y d s \\
& +\int_{r}^{A} \int_{s}^{A}\left|u_{0}(y)\right| d y d s+\int_{r}^{A} \int_{s}^{A}|u(t, y)| d y d s \\
& +\int_{0}^{t} \int_{0}^{s} \int_{r}^{A} \frac{n-1}{y}|u(\tau, y)| d y d \tau d s \\
& \leq \epsilon Q+\frac{1}{4} Q^{p} A^{2}+\frac{1}{4} Q+\frac{1}{4} \frac{n-1}{r_{0}^{2}} A^{2} Q+\frac{1}{2} A^{2} Q+2 A^{2} Q+\frac{1}{4} \frac{n-1}{r_{0}} A Q \\
& =\epsilon Q+\left(\frac{1}{4} Q^{p-1} A^{2}+\frac{1}{4}+\frac{1}{4} \frac{n-1}{r_{0}^{2}} A^{2}+\frac{1}{2} A^{2}+2 A^{2}+\frac{1}{4} \frac{n-1}{r_{0}} A\right) Q \\
& \leq \epsilon Q+(1-\epsilon) Q=Q
\end{aligned}
$$

Now we will estimate $\left(S_{11}(u)\right)_{t}$ for it we have

$$
\begin{aligned}
& \left(S_{11}(u)\right)_{t}=-\epsilon u_{t}+\int_{0}^{t} \int_{r}^{A} \int_{y}^{A} u_{t}^{p} d z d y d \tau d s \\
& +\int_{0}^{t} u(\tau, r) d \tau d s+\int_{0}^{t} \int_{r}^{A} \int_{y}^{A} \frac{n-1}{z^{2}} u(\tau, z) d z d y d \tau d s+\int_{r}^{A} \int_{s}^{A} u_{1}(y) d y d s \\
& -\int_{r}^{A} \int_{s}^{A} u_{t}(t, y) d y d s-\int_{0}^{t} \int_{r}^{A} \frac{n-1}{y} u(\tau, y) d y d \tau d s,
\end{aligned}
$$

from here and from $|u| \leq Q,\left|u_{t}\right| \leq Q, r \in\left[r_{0}, r_{0}+A\right], t \in\left[0, \frac{1}{2}\right]$, the conditions for the constant $A$, we obtain

$$
\begin{aligned}
& \left|\left(S_{11}(u)\right)_{t}\right| \leq \epsilon\left|u_{t}\right|+\int_{0}^{t} \int_{r}^{A} \int_{y}^{A}\left|u_{t}^{p}\right| d z d y d \tau d s \\
& +\int_{0}^{t}|u(\tau, r)| d \tau d s+\int_{0}^{t} \int_{r}^{A} \int_{y}^{A} \frac{n-1}{z^{2}}|u(\tau, z)| d z d y d \tau d s+\int_{r}^{A} \int_{s}^{A}\left|u_{1}(y)\right| d y d s \\
& +\int_{r}^{A} \int_{s}^{A}\left|u_{t}(t, y)\right| d y d s+\int_{0}^{t} \int_{r}^{A} \frac{n-1}{y}|u(\tau, y)| d y d \tau d s \\
& \leq \epsilon Q+\left(\frac{1}{2} A^{2} Q^{p-1}+\frac{1}{2}+\frac{1}{2} \frac{n-1}{r_{0}^{2}} A^{2}+2 A^{2}+\frac{1}{2} A \frac{n-1}{r_{0}}\right) Q \leq \epsilon Q+(1-\epsilon) Q=Q .
\end{aligned}
$$

Consequently

$$
S_{11}: M_{11} \longrightarrow N_{11} \text {. }
$$

From the estimates of $\left|S_{11}(u)\right|$ and $\left|\left(S_{11}(u)\right)_{t}\right|$, follows that if $u \longrightarrow 0, u \in M_{11}$, in the sense of topology of the space $M_{11}$, then $S_{11}(u) \longrightarrow S_{11}(0)$ in the sense of the topology of the space $M_{11}$. Then the operator $S_{11}: M_{11} \longrightarrow M_{11}$ is continuous operator. The set $M_{11}$ is compact space since every sequence of its elements is bounded. Also the set $M_{11}$ is convex.

From Lemma 2.2, Lemma 2.3 and Theorem 2.1 follows that there exists $u^{11} \in$ $M_{11}$ so that $L_{11}\left(u^{11}\right)=S_{11}\left(u^{11}\right)+T_{11}\left(u^{11}\right)$, from here $u^{11}$ is a solution to the problem (2.1), (2.2) and belongs to the space $\mathcal{C}^{2}\left([0,1], \mathcal{C}^{2}\left(\left[r_{0}, r_{0}+A\right]\right)\right)$. 
Now we consider the Cauchy problem

$$
\begin{aligned}
& u_{t t}-\frac{n-1}{r} u_{r}-u_{r r}=u_{t}^{p}, \quad t \in\left[0, \frac{1}{2}\right], r \in\left[r_{0}+A, r_{0}+2 A\right], \\
& u(0, r)=u^{11}(0, r), u_{t}(0, r)=u_{t}^{11}(0, r), \quad r \in\left[r_{0}+A, r_{0}+2 A\right],
\end{aligned}
$$

We define the sets $M_{12}$ and $N_{12}$ as equicontinuous subsets of

$$
\begin{gathered}
\tilde{M}_{12}=\left\{u(t, r) \in \mathcal{C}^{2}\left(\left[0, \frac{1}{2}\right], \mathcal{C}^{2}\left(\left[r_{0}+A, r_{0}+2 A\right]\right)\right),|u(t, r)| \leq Q,\left|u_{t}(t, r)\right| \leq Q,\right. \\
\left.\forall t \in\left[0, \frac{1}{2}\right], r \in\left[r_{0}+A, r_{0}+2 A\right]\right\}, \\
\tilde{N}_{12}=\left\{u(t, r) \in \mathcal{C}^{2}\left(\left[0, \frac{1}{2}\right], \mathcal{C}^{2}\left(\left[r_{0}+A, r_{0}+2 A\right]\right)\right),|u(t, r)| \leq(1+\epsilon) Q,\right. \\
\left.\left|u_{t}(t, r)\right| \leq(1+\epsilon) Q, \forall t \in\left[0, \frac{1}{2}\right], r \in\left[r_{0}+A, r_{0}+2 A\right]\right\},
\end{gathered}
$$

respectively, in these sets we define a norm as follows

$$
\|u\|_{2}=\sup \left\{|u(t, r)|,\left|u_{t}(t, r)\right| \quad t \in\left[0, \frac{1}{2}\right], r \in\left[r_{0}+A, r_{0}+2 A\right]\right\} .
$$

The sets $M_{12}, N_{12}$ are completed normed spaces with this norm.

The operator $L_{12}$ we define with the representation

$$
L_{12}(u)=T_{12}(u)+S_{12}(u),
$$

where

$$
\begin{aligned}
& T_{12}(u)=(1+\epsilon) u \\
& S_{12}(u)=-\epsilon u+\int_{0}^{t} \int_{0}^{s} \int_{r}^{2 A} \int_{y}^{2 A} u_{t}^{p} d z d y d \tau d s \\
& +\int_{0}^{t} \int_{0}^{s} u(\tau, r) d \tau d s+\int_{0}^{t} \int_{0}^{s} \int_{r}^{2 A} \int_{y}^{2 A} \frac{n-1}{z^{2}} u(\tau, z) d z d y d \tau d s+t \int_{r}^{2 A} \int_{s}^{2 A} u_{1}(y) d y d s+ \\
& +\int_{r}^{2 A} \int_{s}^{2 A} u_{0}(y) d y d s-\int_{r}^{2 A} \int_{s}^{2 A} u(t, y) d y d s- \\
& -\int_{0}^{t} \int_{0}^{s} \int_{r}^{2 A} \frac{n-1}{y} u(\tau, y) d y d \tau d s .
\end{aligned}
$$

As in above we conclude that the Cauchy problem (2.6), (2.7) has a solution $u^{12}(t, r) \in \mathcal{C}^{2}\left(\left[0, \frac{1}{2}\right], \mathcal{C}^{2}\left(\left[r_{0}+A, r_{0}+2 A\right]\right)\right)$.

Now we consider the Cauchy problem

$$
\begin{aligned}
& u_{t t}-\frac{n-1}{r} u_{r}-u_{r r}=u_{t}^{p}, \quad t \in\left[0, \frac{1}{2}\right], r \in\left[r_{0}+2 A, r_{0}+3 A\right], \\
& u(0, r)=u^{12}(0, r), u_{t}(0, r)=u_{t}^{12}(0, r), \quad r \in\left[r_{0}+2 A, r_{0}+3 A\right],
\end{aligned}
$$

We define the sets $M_{13}$ and $N_{13}$ as equicontinuous subsets of

$$
\begin{gathered}
\tilde{M}_{13}=\left\{u(t, r) \in \mathcal{C}^{2}\left(\left[0, \frac{1}{2}\right], \mathcal{C}^{2}\left(\left[r_{0}+2 A, r_{0}+3 A\right]\right)\right),|u(t, r)| \leq Q,\left|u_{t}(t, r)\right| \leq Q,\right. \\
\left.\forall t \in\left[0, \frac{1}{2}\right], r \in\left[r_{0}+2 A, r_{0}+3 A\right]\right\} \\
\tilde{N}_{13}=\left\{u(t, r) \in \mathcal{C}^{2}\left(\left[0, \frac{1}{2}\right], \mathcal{C}^{2}\left(\left[r_{0}+2 A, r_{0}+3 A\right]\right)\right),|u(t, r)| \leq(1+\epsilon) Q,\right. \\
\left.\left|u_{t}(t, r)\right| \leq(1+\epsilon) Q, \forall t \in\left[0, \frac{1}{2}\right], r \in\left[r_{0}+2 A, r_{0}+3 A\right]\right\}
\end{gathered}
$$


respectively, in these sets we define a norm as follows

$$
\|u\|_{2}=\sup \left\{|u(t, r)|,\left|u_{t}(t, r)\right| \quad t \in\left[0, \frac{1}{2}\right], r \in\left[r_{0}+2 A, r_{0}+3 A\right]\right\} .
$$

The sets $M_{13}, N_{13}$ are completed normed spaces with this norm.

The operator $L_{13}$ we define with the representation

$$
L_{13}(u)=T_{13}(u)+S_{13}(u),
$$

where

$$
\begin{aligned}
& T_{13}(u)=(1+\epsilon) u \\
& S_{13}(u)=-\epsilon u+\int_{0}^{t} \int_{0}^{s} \int_{r}^{3 A} \int_{y}^{3 A} u_{t}^{p} d z d y d \tau d s \\
& +\int_{0}^{t} \int_{0}^{s} u(\tau, r) d \tau d s+\int_{0}^{t} \int_{0}^{s} \int_{r}^{3 A} \int_{y}^{3 A} \frac{n-1}{z^{2}} u(\tau, z) d z d y d \tau d s+t \int_{r}^{3 A} \int_{s}^{3 A} u_{1}(y) d y d s+ \\
& +\int_{r}^{3 A} \int_{s}^{3 A} u_{0}(y) d y d s-\int_{r}^{3 A} \int_{s}^{3 A} u(t, y) d y d s- \\
& -\int_{0}^{t} \int_{0}^{s} \int_{r}^{3 A} \frac{n-1}{y} u(\tau, y) d y d \tau d s .
\end{aligned}
$$

As in above we conclude that the Cauchy problem (2.8), (2.9) has a solution $u^{13}(t, r) \in \mathcal{C}^{2}\left(\left[0, \frac{1}{2}\right], \mathcal{C}^{2}\left(\left[r_{0}+2 A, r_{0}+3 A\right]\right)\right)$ and etc. Then the function

$$
u^{1}= \begin{cases}u^{11} & t \in\left[0, \frac{1}{2}\right], r \in\left[r_{0}, r_{0}+A\right], \\ u^{12} & t \in\left[0, \frac{1}{2}\right], r \in\left[r_{0}+A, r_{0}+2 A\right], \\ \ldots & \end{cases}
$$

is a solution to the Cauchy problem

$$
\begin{aligned}
& u_{t t}-\frac{n-1}{r} u_{r}-u_{r r}=u_{t}^{p}, \quad t \in\left[0, \frac{1}{2}\right], r \geq r_{0}, \\
& u(0, r)=u_{0}(r), u_{t}(0, r)=u_{1}(r), \quad r \geq r_{0},
\end{aligned}
$$

which belongs to the space $\mathcal{C}^{2}\left(\left[0, \frac{1}{2}\right], \mathcal{C}^{2}\left(\left[r_{0}, \infty\right)\right)\right)$. We note that in above definition of the solution $u^{1}$ we agglutinate continuous - differentiable $u^{11}$ and $u^{12}$ in $r=$ $r_{0}+A, u^{12}$ and $u^{13}$ in $r=r_{0}+2 A$ and etc. if it is necessary.

Now we consider the Cauchy problem

$$
\begin{aligned}
& u_{t t}-\frac{n-1}{r} u_{r}-u_{r r}=u_{t}^{p}, \quad t \in\left[\frac{1}{2}, 1\right], r \in\left[r_{0}, r_{0}+A\right], \\
& u\left(\frac{1}{2}, r\right)=u^{1}\left(\frac{1}{2}, r\right), u_{t}\left(\frac{1}{2}, r\right)=u_{t}^{1}\left(\frac{1}{2}, r\right), \quad r \in\left[r_{0}, r_{0}+A\right],
\end{aligned}
$$

We define the sets $M_{21}$ and $N_{21}$ as equicontinuous subsets of

$$
\begin{aligned}
& \tilde{M}_{21}=\left\{u(t, r) \in \mathcal{C}^{2}\left(\left[\frac{1}{2}, 1\right], \mathcal{C}^{2}\left(\left[r_{0}, r_{0}+A\right]\right)\right),|u(t, r)| \leq Q,\left|u_{t}(t, r)\right| \leq Q,\right. \\
& \left.\forall t \in\left[\frac{1}{2}, 1\right], r \in\left[r_{0}, r_{0}+A\right]\right\},
\end{aligned}
$$




$$
\begin{aligned}
& \tilde{N}_{21}=\left\{u(t, r) \in \mathcal{C}^{2}\left(\left[\frac{1}{2}, 1\right], \mathcal{C}^{2}\left(\left[r_{0}, r_{0}+A\right]\right)\right),|u(t, r)| \leq(1+\epsilon) Q,\right. \\
& \left.\left|u_{t}(t, r)\right| \leq(1+\epsilon) Q, \forall t \in\left[\frac{1}{2}, 1\right], r \in\left[r_{0}, r_{0}+A\right]\right\}
\end{aligned}
$$

respectively in these sets we define a norm as follows

$$
\|u\|_{2}=\sup \left\{|u(t, r)|,\left|u_{t}(t, r)\right| \quad t \in\left[\frac{1}{2}, 1\right], r \in\left[r_{0}, r_{0}+A\right]\right\} .
$$

The sets $M_{21}, N_{21}$ are completed normed spaces with this norm.

The operator $L_{21}$ we define with the representation

$$
L_{21}(u)=T_{21}(u)+S_{21}(u),
$$

where

$$
\begin{aligned}
& T_{21}(u)=(1+\epsilon) u, \\
& S_{21}(u)=-\epsilon u+\int_{\frac{1}{2}}^{t} \int_{\frac{1}{2}}^{s} \int_{r}^{A} \int_{y}^{A} u_{t}^{p} d z d y d \tau d s \\
& +\int_{\frac{1}{2}}^{t} \int_{\frac{1}{2}}^{s} u(\tau, r) d \tau d s+\int_{\frac{1}{2}}^{t} \int_{\frac{1}{2}}^{s} \int_{r}^{A} \int_{y}^{A} \frac{n-1}{z^{2}} u(\tau, z) d z d y d \tau d s \\
& +\left(t-\frac{1}{2}\right) \int_{r}^{A} \int_{s}^{A} u_{1}(y) d y d s+ \\
& +\int_{r}^{A} \int_{s}^{A} u_{0}(y) d y d s-\int_{r}^{A} \int_{s}^{A} u(t, y) d y d s- \\
& -\int_{\frac{1}{2}}^{t} \int_{\frac{1}{2}}^{s} \int_{r}^{A} \frac{n-1}{y} u(\tau, y) d y d \tau d s .
\end{aligned}
$$

As in above we conclude that the Cauchy problem (2.10), (2.11) has a solution $u^{21}(t, r) \in \mathcal{C}^{2}\left(\left[\frac{1}{2}, 1\right], \mathcal{C}^{2}\left(\left[r_{0}, r_{0}+A\right]\right)\right)$ and etc., the function

$$
u^{2}= \begin{cases}u^{21} & t \in\left[\frac{1}{2}, 1\right], r \in\left[r_{0}, r_{0}+A\right], \\ u^{22} & t \in\left[\frac{1}{2}, 1\right], r \in\left[r_{0}+A, r_{0}+2 A\right], \\ \cdots & \end{cases}
$$

is a solution to the Cauchy problem

$$
\begin{aligned}
& u_{t t}-\frac{n-1}{r} u_{r}-u_{r r}=u_{t}^{p}, \quad t \in\left[\frac{1}{2}, 1\right], r \geq r_{0}, \\
& u\left(\frac{1}{2}, r\right)=u^{1}\left(\frac{1}{2}, r\right), u_{t}\left(\frac{1}{2}, r\right)=u_{t}^{1}\left(\frac{1}{2}, r\right), \quad r \geq r_{0},
\end{aligned}
$$

which belongs to the space $\mathcal{C}^{2}\left(\left[\frac{1}{2}, 1\right], \mathcal{C}^{2}\left(\left[r_{0}, \infty\right)\right)\right)$ and etc. We note that in above definition of the solution $u^{2}$ we agglutinate continuous - differentiable $u^{21}$ and $u^{22}$ in $r=r_{0}+A, u^{22}$ and $u^{23}$ in $r=r_{0}+2 A$ and etc. if it is necessary. The function

$$
u= \begin{cases}u^{1} \quad t \in\left[0, \frac{1}{2}\right], r \geq r_{0}, \\ u^{2} \quad t \in\left[\frac{1}{2}, 1\right], r \geq r_{0}, \\ \cdots\end{cases}
$$

is a solution to the Cauchy problem (1.1), (1.2) which belongs to the space

$$
\mathcal{C}^{2}\left([0, \infty), \mathcal{C}^{2}\left(\left[r_{0}, \infty\right)\right)\right) .
$$




\section{Acknowledgemets}

The author is partially supported by research grant of Faculty of Mathematics and Informatics, Sofia University, Bulgaria, with chief Associate Professor Angel Zhivkov.

\section{References}

[1] Georgiev, S. Blow up of the solutions of nonlinear parabolic equation, Dynamics od PDE, 2009, Vol. 1.

[2] Grillakis, M. Regularity for the wave equation with a critical nonlinearity, Comm. Pure Appl. Math. 45, 1992, pp. 749-774.

[3] Jörgens, K. Das Anfangswertproblem im Grossen für eine Klasse nicht-linear Wellengleichungen, Math. Z., 77, 1961, pp. 295-308.

[4] Krasnosel'skii, M., P.Zabrejko, Geometrical Methods of Nonlinear Analysis, Springer Verlag, Series 263, 1984.

[5] Segal, I. The global Cauchy problem for a relativistic scalar field with power interaction, Bull. Soc. Math, France 91, 1963, pp. 129-135.

[6] Shatah, J., M. Struwe. Geometric wave equation. Courant lecture notes in mathematics 2(1998).

[7] Sideris, T. Global behaviour of solutions to nonlinear wave equations in three space dimensions, Comm. in PDE, 8(12)(1983), 1291-1323.

[8] Xiang, T., Rong Yuan. A class of expansive - type Krasnosel'skii fixed point theorems. Nonlinear Analysis, 71(2009), 3229-3239.

Department of Differential Equations, University of Sofia, Sofia, Bulgaria E-mail address: sgg2000bg@yahoo.com 